\title{
The emergence of a coherent structure for coherent structures: localized states in nonlinear systems
}

\author{
By J. H. P. DAWES \\ Department of Mathematical Sciences, University of Bath, Claverton Down, Bath \\ $B A 27 A Y, U K$
}

Coherent structures emerge from the dynamics of many kinds of dissipative, externally driven, nonlinear systems, and continue to provoke new questions that challenge our physical and mathematical understanding.

In one specific sub-class of such problems, where a pattern-forming, or 'Turing', instability occurs, rapid progress has been made recently in our understanding of the formation of localized states: patches of regular pattern surrounded by the unpatterned homogeneous background state.

This short review article surveys the progress that has been made for localized states, proposes three areas of application for these ideas that would take the theory in new directions and ultimately be of substantial benefit to areas of applied science. Finally I offer speculations for future work, based on localized states, that may help researchers to understand coherent structures more generally.

Keywords: pattern formation, Turing instability, bifurcation, homoclinic snaking

\section{Introduction}

Encouraged by the success of low-dimensional dynamical systems theory in the 1980s in explaining the origin of complicated behaviour in nonlinear ordinary differential equations, researchers in what might be called 'nonlinear science' are attempting to carry through a similar programme for spatially-extended systems of many kinds. In this review I will restrict attention to systems that are internally dissipative and externally driven; 'nonequilibrium' in the physics terminology. More precisely, such systems (for example chemical and biological kinetics, viscous fluid mechanics, frictional solid mechanics) equilibrate at a level of activity sufficient to provide a global energy balance. Commonly this involves the formation of spatial structure with a single well-defined lengthscale. The bifurcations in which such a patterned state is born out of a homogeneous state are known, naturally enough, as spontaneous pattern-forming, or 'Turing' instabilities (Turing 1952). Spontaneity is important here: the external driving is still imposed in a spatially uniform fashion, so the emergence of structure is a clear symmetry-breaking transition.

Well-known examples of spontaneous pattern formation include Rayleigh-Bénard convection, in a layer of viscous fluid heated from below, Faraday surface waves on a the surface of a vertically shaken liquid or granular layer, and reaction-diffusion dynamics, proposed for example to organise many processes in developmental biology (Cross \& Hohenberg 1993; Murray 2003; Hoyle 2006). 
The theoretical analysis of 'pattern-forming instabilities' of these kinds in fluid mechanical problems can be traced back to the early 20th century in the case of Rayleigh-Bénard convection (Rayleigh 1916). The subsequent emergence of mathematical biology as a distinct area within applied mathematics has meant that Alan Turing's demonstration (Turing 1952) that such patterns could result from a more general, and biologically-relevant, mechanism of local activation coupled with longer-range inhibition has continued to have a significant impact in shaping our understanding of mechanisms for morphogenesis.

Turing's original observation, that spontaneous pattern formation may arise through a linear instability due to coexisting diffusive effects that operate on sufficiently different spatial scales even when the homogeneous state is stable in the absence of diffusion, has been made mathematically precise, and forms the basis for arguments about the generic nature of such instabilities and the typical patterns which result.

More recently, it has become clear that a pattern-forming or 'Turing' instability can also result in the formation of spatially localized states, even if the system remains driven in a spatially uniform fashion. These localized states resemble a number of periods of the periodic pattern that we might expect, but surrounded by the spatially uniform background state rather than extending to fill the whole spatial domain. The two key ingredients for the formation of localized states near Turing instabilities are (i) bistability, and (ii) pinning.

Bistability means that the spatially uniform state and the patterned state are stable to small disturbances over a single range of values of the system parameters. In the language of bifurcation theory, bistability occurs when the Turing instability is subcritical, creating a small-amplitude unstable pattern which exists alongside a larger amplitude stable pattern as well as the stable trivial unpatterned state.

Pinning refers, by analogy with the motion of defects in crystals, to the local energy well in which the localized state sits: there is an energetic barrier to overcome in order either for the localized patch of pattern to propagate further into the surrounding background state, or conversely, for the background state to be able to swamp the localized state and remove it. In spatially continuous systems, described by partial differential equations, the periodicity of the pattern itself provides this pinning effect: pinning is therefore a generic feature for these systems. In spatially discrete dissipative systems, where the discrete nature of the system is modelled in ways that are closely analogous to those used in atomic lattices (such as the Frenkel-Kontorova model), the discrete nature of the system generates pinning also in a generic fashion.

Recent mathematical work has proved that localized states arise generically near Turing instabilities, and has greatly clarified their existence and bifurcations in model equations such as the $1 \mathrm{D}$ bistable Swift-Hohenberg equation:

$$
u_{t}=r u-\left(1+\partial_{x}^{2}\right)^{2} u+N(u ; s)
$$

(Swift \& Hohenberg 1977) where $u(x, t)$ is a scalar variable and $r<0<s$ are parameters, and $N(u ; s)$ refers to the choice of nonlinear terms that give rise to the subcritical bifurcation of small amplitude states and subsequence re-stabilisation of the dynamics at larger amplitudes. Popular choices are $N_{1}(u ; s)=s u^{2}-u^{3}$ and $N_{2}(u ; s)=s u^{3}-u^{5}$. The resulting bifurcation structure of localized states has a characteristic structure containing two intertwining curves of solutions and as a 
result the process of formation of these families of localized states is frequently referred to as 'homoclinic snaking'. While it is not a normal form in the strict sense, (1.1) is often taken in the literature as a canonical model equation for homoclinic snaking, and recent investigation through a combination of numerical and analytic approaches supports its employment as a generic model equation.

The structure of this review is as follows. In section $2 \mathrm{I}$ list physical systems in which localized states have been observed, either in laboratory or numerical experiments, beforel discussing analytical approaches to the dynamics of (1.1). Section 3 presents three areas in which both bistability and pinning effects are likely to be present and hence the theory of localized states should be an important part of our understanding of the problem. Conclusions and wilder speculations for future research directions are contained in section 4 .

\section{Recent progress}

In this section I first summarise a number of areas in which localized states have been observed, either in laboratory experiments, or in theoretical modelling work (section 2-ian). Then I very briefly outline the dynamics of the canonical 1D SwiftHohenberg model (section 2b) , before discussing additional issues.

\section{(a) Motivations}

Localized patterns have been described in experiments and models in an extremely wide variety of fields. The literature on the localisation of buckling patterns of elastic beams and shells is particularly rich (Potier-Ferry 1983, Hunt et al. 2000); localized states have also been analysed in fluid mechanics (in particular in doubly-diffusive convection problems (Riecke \& Granzow 1999; Riecke 1999; Batiste \& Knobloch 2005) and magnetoconvection (Blanchflower 1999; Dawes 2007, 2008); nonlinear optics (Akhmediev \& Ankiewicz 2005); gas discharge systems (Purwins et al. 2005); vertically oscillated granular and viscoelastic media (Umbanhowar et al. 1996, Lioubashevski et al. 1999); ferrofluid instability (Richter \& Barashenkov 2005), surface catalysis, mathematical neuroscience, developmental biology and many others.

The study of localized states is therefore of fundamental importance to research in all these fields. However, it is only recently that the mathematical structure and organisation of localized states has become completely understood even in the simplest, one-dimensional, case. Alongside this mathematical structure, recent work has exploited the existence of numerical continuation packages such as AUTO (Doedel 2007). The resulting bifurcation diagrams that can be compiled clarify hugely the organisation, existence, and stability of localized patterns and have contributed to a resurgence of interest in the area.

\section{(b) The Swift-Hohenberg model}

The simplest pattern-formation situation in which localized states appear is given by considering a single scalar PDE for a quantity $u(x, t)$ that is posed on the real line $-\infty<x<\infty$. We assume that the trivial state $u(x, t) \equiv 0$ exists for all parameter values and is linearly stable when a parameter $r$ is negative. We 
further assume that it first loses stability, at $r=0$, to Fourier modes $\mathrm{e}^{\mathrm{i} k x}$ with $k$ near unity. We take the PDE to be first-order in time and left-right reflection symmetric, i.e. unchanged under the operation $(x, u) \rightarrow(-x, u)$. This latter condition (a 'reversibility') implies that terms in the PDE contain even numbers of $x$-derivatives. The simplest canonical model equation with these properties is the cubic-quintic fourth-order Swift-Hohenberg equation

$$
u_{t}=r u-\left(1+\partial_{x}^{2}\right)^{2} u+s u^{3}-u^{5} .
$$

For $s>0$ the instability of the state $u(x, t)=0$ at $r=0$ is to modes $\sim \mathrm{e}^{\mathrm{k} k x}$ with $k$ near unity, and this instability is subcritical. As a result, there is a region of bistability between stable large-amplitude space-periodic solutions and the trivial solution $u=0$, see figure 1(a).

Equation (2.1) can be analysed from two points of view. One is the asymptotic reduction of (2.1) to a Ginzburg-Landau equation via a multiple-scales expansion. The second is to consider equilibria of the fourth-order spatial dynamical system in $x$ given by setting $u_{t}=0$ in which localized states correspond to trajectories homoclinic to $u=0$.

\section{(i) Asymptotics}

In the Ginzburg-Landau approach near the codimension-two point where $r=$ $s=0$ it is appropriate to introduce the scaled variables $\mu$ and $\hat{s}$ defined by $r=\varepsilon^{2} \mu, s=\varepsilon^{2} \hat{s}$, and the long length and time scales $X=\varepsilon^{2} x$ and $T=\varepsilon^{4} t$. We look for steady solutions to (2.1) perturbatively with the multiple-scales expansion $u(x, t)=\varepsilon\left(A(X, T) \mathrm{e}^{1 x}+\right.$ c.c. $)+\varepsilon^{2} u_{2}(x, X, t, T)+O\left(\varepsilon^{3}\right)$ where c.c. denotes the complex conjugate. We substitute this ansatz into (2.1) and solve at successive orders in $\varepsilon$, applying a 'solvability condition' at each order to eliminate secular terms that would otherwise disrupt the asymptotic ordering of the solution in powers of $\varepsilon$. From the solvability condition at $O\left(\varepsilon^{5}\right)$ we deduce the cubic-quintic GinzburgLandau equation for the complex-valued envelope $A(X, T)$ :

$$
A_{T}=\mu A+4 A_{X X}+3 \hat{s} A|A|^{2}-10 A|A|^{4} .
$$

Two distinct constant solutions $A=A_{0}^{ \pm}$exist for $-9 \hat{s}^{2} / 40<\mu<0$ with the smaller (larger) amplitude state being unstable (stable), respectively. These correspond to exactly periodic solutions for $u(x)$, see figure 1(b).

There are also non-constant solutions which correspond to localized states: At small amplitudes $|A| \ll 1$ the balance between the first three terms on the righthand side of (2.2) indicates that non-constant equilibrium solutions also exist, with a profile that is close to a 'sech' function. As $\mu$ decreases, the solution profile broadens and more closely resembles a pair of 'tanh'-like fronts connecting the constant solutions $A=A_{0}^{+}$and $A=0$. At the Maxwell point $\mu=-27 \hat{s}^{2} / 160$ these two constant solutions are energetically equal (note that equations (2.2) and (2.1) are variational) and so a front between them remains stationary. Hence it looks possible that stationary solutions for $u(x)$ can be constructed which consist of a patch of almost uniform pattern surrounded by the trivial state $u=0$. That this indeed occurs rests on Pomeau's observation (Pomeau 1986) that such a front between the periodic pattern and the trivial solution $u=0$ is pinned by the periodicity 


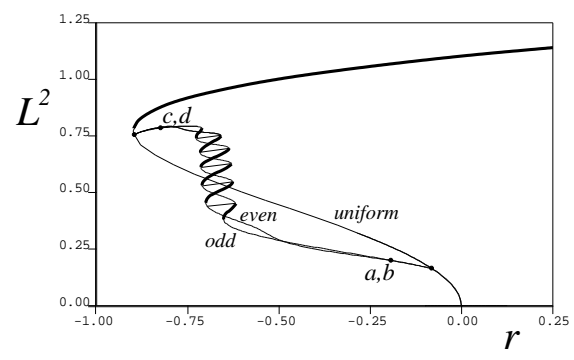

(a)

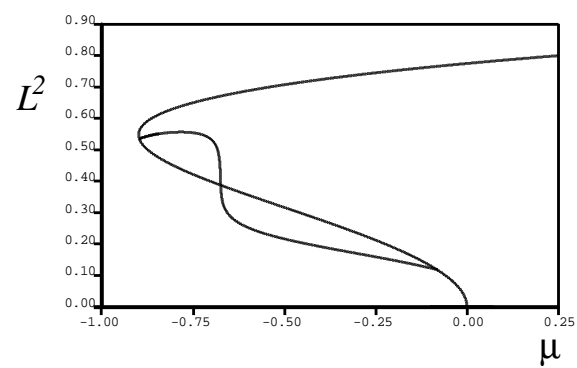

(b)

Figure 1. Bifurcation diagrams for (a) the Swift-Hohenberg equation (2.1) solved in the domain $0 \leq x \leq L=10 \pi$ and (b) the Ginzburg-Landau equation (2.2) solved in the domain $0 \leq X \leq 10 \pi$, both using periodic boundary conditions. The vertical line segment of the curve in (b) indicates the Maxwell point $\mu_{m x}$ around which the snaking curves in (a) open up and intertwine.

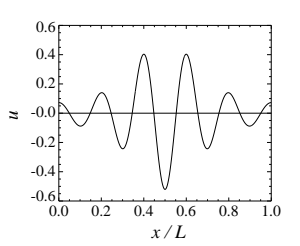

(a)

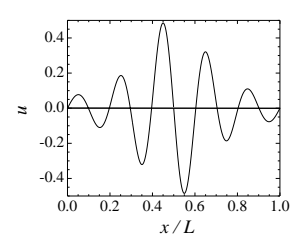

(b)

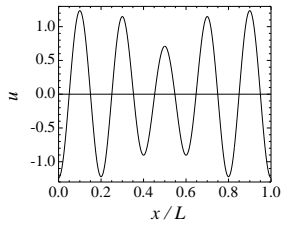

(c)

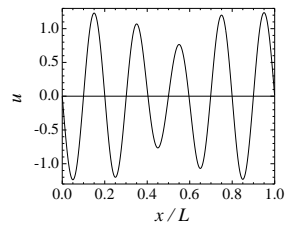

(d)

Figure 2. Localized states on the odd and even snaking curves shown in figure 1(a), corresponding to the labels $a, b, c$ and $d .(a)$ and $(c)$ lie on the even-symmetric branch and $(b)$ and $(d)$ on the odd-symmetric branch.

of the pattern itself. The pinning effect emerges through the interaction of the long-wavelength envelope scale $X$ and the original pattern scale $x$. Since these are decoupled at every order in the multiple-scales perturbation theory, this interaction must necessarily be a 'beyond-all-orders' effect. The relevant exponentially small terms, discussed qualitatively by many authors, have only recently been calculated correctly (Chapman \& Kozyreff 2009). Pinning expands the region of existence of the localized states from a single line into a cusp-shaped wedge in the $(r, s)$ plane. Within the cusp-shaped pinning region the two curves of localized states intertwine in a characteristic fashion which gives rise to the term 'homoclinic snaking', as shown in figure 1(a). For the cubic-quintic Swift-Hohenberg equation (2.1) localized states on the two curves are odd and even respectively, as illustrated in figure 2 . The short horizontal lines in figure 1(a) between the snaking curves represent 'ladder' branches of asymmetric (i.e. neither odd nor even) localized states.

\section{(ii) Spatial dynamics}

A different approach to (1.1) is to neglect the time derivative and take the spatial coordinate to be the time-like evolution variable; this framework is therefore often referred to as 'spatial dynamics'. In this framework the pattern-forming instability that occurs at $r=0$ is, due to the reversibility, a Hamiltonian-Hopf bifurcation 
(a.k.a. a 1 : 1 resonance). The normal form analysis of this Hamiltonian-Hopf bifurcation was carried out by Iooss \& Perouème (1993) and later extended by Woods \& Champneys (1999), Coullet et al (2000) and Burke \& Knobloch (2006); taken together, these papers establish many features of the bifurcation problem, for example the existence in the normal form of small-amplitude localized states near the bifurcation point and that two of these states are then guaranteed to exist along bifurcating solution branches away from $r=0$ : it is these branches that correspond to the commonly identified localized patterns. The characteristic intertwined wiggling of the snaking branches arises from the generic behaviour of the unstable and stable manifolds of the origin as they pass through a heteroclinic tangle.

In the resulting bifurcation diagram the secondary branches are sometimes referred to as 'ladders'. That such asymmetric branches should exist generically can be deduced both from a general bifurcation-theoretic approach (Beck et al. 2009) and deduced from the exponential asymptotics results of Chapman \& Kozyreff (2009).

\section{(c) Effects of a finite domain}

The 'spatial dynamics' approach to homoclinic snaking necessarily deals with homoclinic orbits: solutions on the real line. Practical applications, though, necessarily demand finite domains and suitable boundary conditions. Numerical investigation of the Swift-Hohenberg equation in finite domains (Bergeon et al. 2008, Dawes 2009) illustrates how the homoclinic snaking persists when the domain is large and finite, and how the snake winds up or unravels as the domain size increases or decreases, respectively.

As illustrated by figure 1(a), in a finite domain the snaking curves bifurcate directly from the periodic pattern close to, but not exactly at, $r=0$. As the localized state expands to fill the domain it ceases to be localised and eventually the snaking curve reconnects to the periodic pattern near the saddle-node point on the periodic branch. The details of this reconnection are more complicated than one might perhaps expect, particularly when a second parameter, for example the domain size $L$, is allowed to vary. It appears that the reconnection of the snaking branch is organised by a mode interaction between the $n$-roll and $n+1$-roll branches of periodic patterns which move close to each other as $L$ increases from $2 \pi n$ to $2 \pi(n+1)$ (Dawes, 2009).

\section{(d) Large-scale modes}

In a subset of the application areas listed in section 2 it has been realised that the pattern-forming instability is coupled to a large-scale mode which is neutrally stable at long wavelengths. Such a mode arises naturally in some situations due to a conservation law (Matthews \& Cox 2000), and cannot be ignored in a weakly nonlinear analysis. The effect of such a neutral mode is to 'stretch out' the homoclinic snaking in parameter space, in a way that can be captured by a more subtle asymptotic analysis that leads naturally to Ginzburg-Landau-type equations that contain nonlocal terms. The large-scale field causes stable localized states to exist over a greater parameter range than just near the Maxwell point, and in many cases localized 


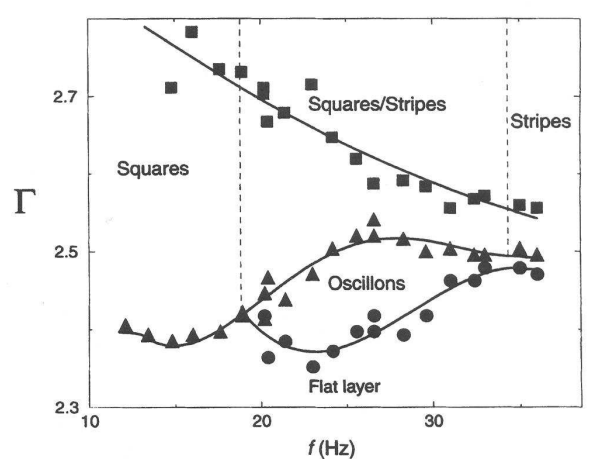

(a)

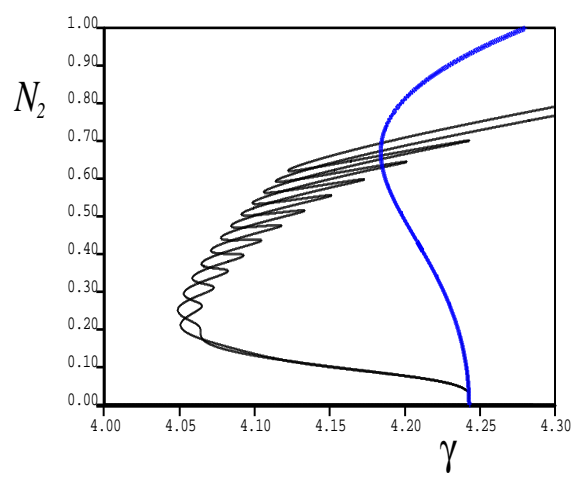

(b)

Figure 3. (a) Experimentally-determined regime diagram from Umbanhowar et al. (1996) showing that oscillons (i.e. localized states), which exist only in the 'bubble', exist more subcritically than extended patterns which only exist above the triangles. $\Gamma$ is the dimensionless acceleration (driving parameter) and $f$ is the frequency of the sinusoidal vertical motion of the granular layer. Copyright Nature Publishing Group (1996). (b) Bifurcation diagram for slanted snaking in the oscillon model (Dawes \& Lilly 2009) showing localized states (intertwined thin black curves) existing for $\gamma(\sim \Gamma)$ more negative than patterned states (thick blue curve), corresponding to (b) around $f=25 \mathrm{~Hz}$. The vertical axis $N_{2}$ is a solution norm.

states exist more subcritically than might be expected, as illustrated in figure 3 (b). Recent work (Dawes 2007, 2008; Dawes \& Lilley 2009) provides both a physical and a mathematical mechanism that resolves differences between the traditional homoclinic snaking bifurcation diagram (figure 1a) and the 'slanted snaking' that arises in the large-scale-mode case. Intruigingly, similar mechanisms operate in several different application areas: models for magnetoconvection (Dawes 2007, 2008), and nonlinear optics (Firth et al. 2007), and experimental results obtained both for dielectric gas discharge (H.-G. Purwins, unpublished) and for vertically vibrated layers of granular material, see Umbanhowar et al. (1996) from which figure 3(a) is reproduced. Asymptotic analysis of a model problem (proposed by Tsimring \& Aranson 1997) for the vertically shaken granular layer case shows (figure $3 \mathrm{~b}$ ), in agreement with experimental results (figure 3a), that the localized states can exist more subcritically than the uniform periodic pattern. The uniform periodic pattern is indicated by the thick blue line in figure 3(b) and bifurcates subcritically before turning around at finite amplitude, but the snaking curves are not constrained to lie only between the linear instability and the saddle-node point at the leftmost extreme of the blue curve: they appear at lower values of the bifurcation parameter $\gamma$ due to the large-scale mode.

The physical mechanism for this difference is a balance between diffusion of the large-scale quantity and nonlinear gradients of excitation of activity. In the vertically shaken layers, conservation of mass leads to a diffusion equation with a nonlinear term indicating that mass is ejected from more active regions in the layer. This positive feedback stabilises oscillons since this nonlinear expulsion of material can balance diffusion even below the linear stability threshold. In a suitable 

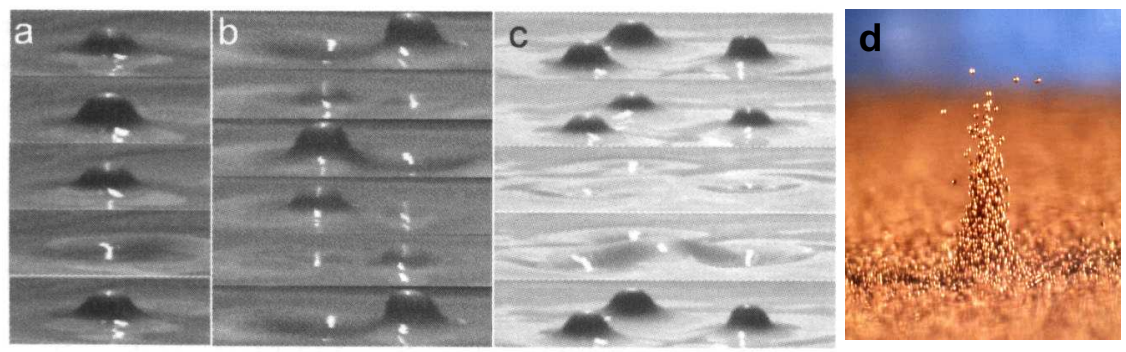

Figure 4. Experimentally observed oscillons in layers of vertically shaken material: (a), (b), (c) in a viscoelastic clay suspension of density $\rho=1.28 \mathrm{~g} / \mathrm{cm}^{3}$; (a) shows a single oscillon at $f=14 \mathrm{~Hz}$, (b) an oscillon pair at $f=20 \mathrm{~Hz}$, (c) an oscillon triad at $f=25 \mathrm{~Hz}$. Frames are equally spaced in time and cover two periods of the vertical forcing, showing the subharmonic nature of the localised response (Lioubashevski et al 1997). (d) Sideview of a localised state in a layer of bronze spheres of diameter $\approx 0.16 \mathrm{~mm}$, approx 17 particles deep (Umbanhowar et al 1996). Again, the response is subharmonic: after one period of the forcing the peak will have collapsed to a crater, re-forming a peak after a second period of the forcing has passed. (a) - (c) are reprinted with permission from: Lioubashevski et al (1999) and are copyright (1999) by the American Physical Society. (d) is copyright Nature Publishing Group (1996).

asymptotic limit this balance can be sustained even at small amplitude, and hence a modified weakly nonlinear analysis is possible.

\section{Applications of localized states}

In this section I offer three applications in which the theory of localized states may prove extremely useful.

The general philosophical point is that many climate change problems, illustrated here by the examples of ocean circulations and desertification, involve spatially extended systems which evolve smoothly until a critical parameter value is reached where catastrophic and irreversible change occurs: this is the idea of a tipping point. In the language of nonlinear dynamics, this corresponds to the existence of a saddle-node bifurcation, together with hysteresis (arising from bistability) so that after the catastrophic change there is no simple, reversible path by which to recover the original climate state. This is the kind of system in which localized states exist; therefore it appears ambitious but reasonable to suggest that properties of localized states could form the basis of diagnostic tests for proximity to tipping points. For example, a local perturbation of the system into the undesirable state produces a front between parts of the system that are locally in the two different stable states. We then can understand, quantitatively, how the front will generically move in order to eliminate the non-uniformity. Far from the tipping point one would expect the local perturbation into the undesirable state to vanish, while closer to the tipping point (and past the 'Maxwell point' for the system where a front between the two states would be stationary) the undesirable state would win. In this way, properties of the front (e.g. its velocity) could be used to predict the distance from the current system state to the 'Maxwell point' and hence to the saddle-node bifurcation (tipping point). 
(a) Transition to turbulence in shear flows

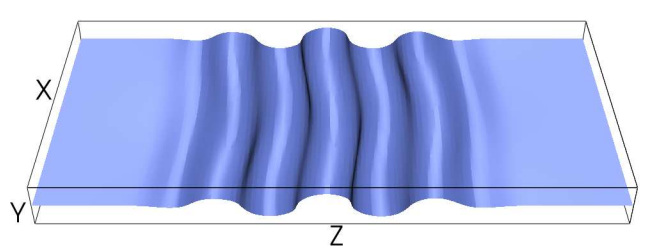

(a)

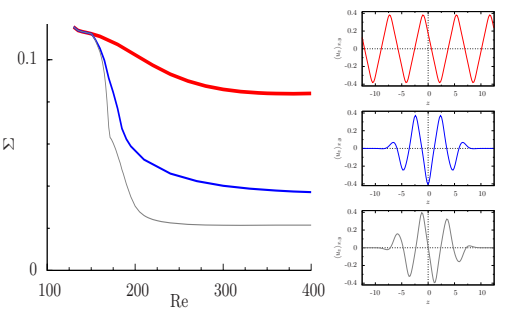

(b)

Figure 5. (a) Surface of vanishing streamwise velocity $u$ for an unstable steady localized state in plane Couette flow $\mathbf{u} \equiv(u, v, w)=(y, 0,0)$ between stress-free surfaces at $y= \pm 1$, where $(u, v, w)$ are the velocity components in, respectively, the $x$ (streamwise), $y$ (wall-normal) and $z$ (spanwise) directions. This very closely resembles the middle inset figure in (b) since Couette flow is symmetric under $y \rightarrow-y$. Reproduced with permission from Schneider, Marinc \& Eckhardt (2009a). (b) Typical bifurcation diagram of localized (blue, black) and spanwise-periodic (red) states, courtesy of Tobias Schneider. Note the similarity of the lower two inset figures to figure 2(a), (b).

One classic example of bistability arises in the transition to turbulence from simple shear flows, for example, plane Couette flow. In plane Couette flow a transition from laminar flow, to persistent turbulence is observed experimentally and computationally in a statistically reliable fashion with increasing Reynolds number, without the existence of a linear instability of the laminar state. Exciting recent work on this problem of 'transition to turbulence' has uncovered localized states which appear to play important roles in the structure of the collection of 'edge states' - unstable flow structures that separate the basins of attraction of the laminar state and the persistently turbulent one (Schneider, Marinc \& Eckhardt 2009; Schneider, Gibson \& Burke 2010). Understanding the details of the role of localized states is an exciting development which will change and vastly clarify our understanding of fluid flow at moderate Reynolds numbers.

\section{(b) The Atlantic meridional overturning circulation (MOC)}

The Contribution of Working Group I to the Fourth Assessment Report of the Intergovernmental Panel on Climate Change (IPCC), Bindoff et al (2007), discusses the importance of the MOC at length, noting the difficulties associated with obtaining reliable data and calibrating ocean circulation models for the MOC. The IPCC report also affirms the influence of the MOC on global climate, establishing its important role in climate dynamics, stating clearly for example (chapter 5, page 397) that There is evidence for a link between the MOC and abrupt changes in surface climate during the past $120 \mathrm{kyr} .$. It is certainly clear that the MOC is a crucial determinant of European climate since it transports heat from equator to pole, and this is estimated to keep the Atlantic around $4^{\circ} \mathrm{C}$ warmer than it would otherwise be.

Figure 6(a) shows a bifurcation diagram for the MOC computed numerically directly from a global ocean model (Huisman et al 2009). The bistability it shows 

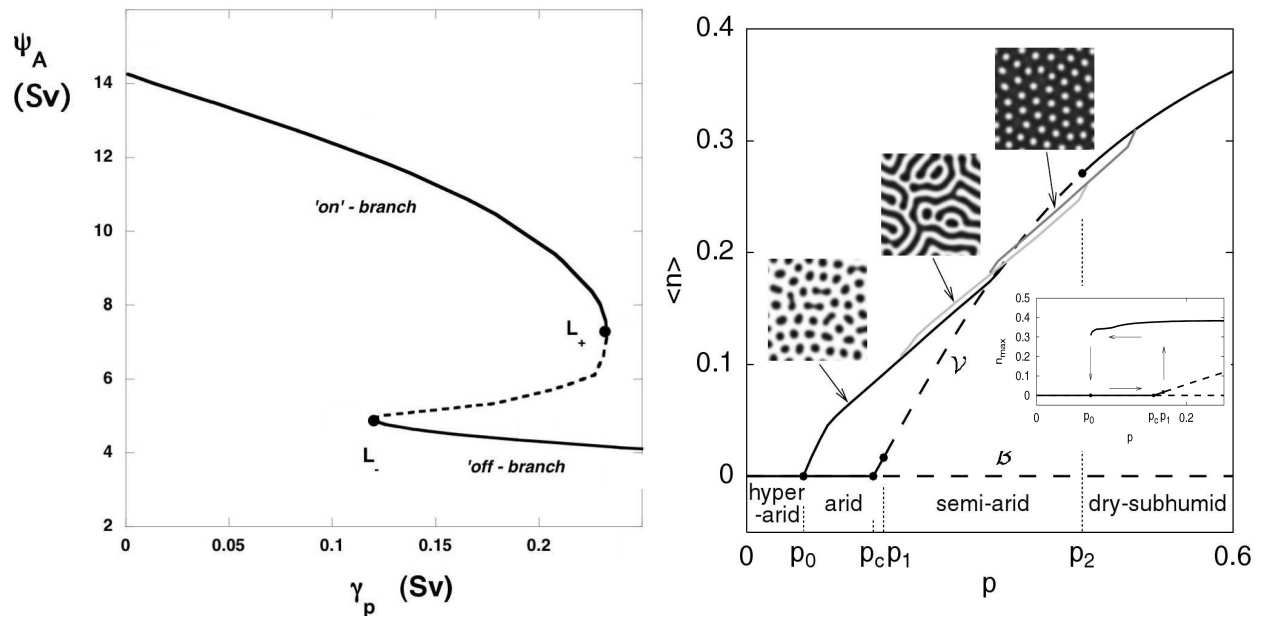

Figure 6. (a) Bifurcation diagram for the strength of the Atlantic meridional overturning circulation $\psi_{A}$ as the anomalous freshwater forcing parameter $\gamma_{p}$ is varied. Reproduced with permission from Huisman et al. (2009). Sv refers to the Sverdrup, a unit of volume flux: $1 S v=10^{6} \mathrm{~m}^{3} \mathrm{~s}^{-1}$. (b) Bifurcation diagram for the desertification model (3.2) (3.3) proposed by von Hardenberg et al (2001) showing numerically-determined ranges of existence of different patterns, and (insert) bistability at low precipitation rates in the range $p_{0}<p<p_{1}$.

is common to many classes of such model, originating with Stommel (1961), and is well known (see for example Launder \& Thompson 2008). Models suggest that an increasing flux of freshwater from melting polar ice (i.e. increasing $\gamma_{p}$ ) pushes the MOC towards the limit point $L_{+}$which, if reached, would result in the MOC switching off abruptly, and the European climate therefore cooling.

Simplifying the geometry of the Atlantic ocean to a fluid-filled rectangular box, one can generate very similar flows in the laboratory (Whitehead 1996). The equatorial region is modelled by a heated plate attached to one side of the box, providing a constant source of hot, and also salt-enriched, water. This hot saline water flows across the top of the box to the opposite ('polar') side where a stream of colder fresh water is introduced. The cold fresh water cools the hot stream on contact, which forces it to sink since its salinity makes it now denser than its surroundings. Hence a return flow from pole to equator is formed at depth in the container. Throwing away the geometry and almost all the mechanics, one can treat the problem as consisting of flows between two separate boxes of fluid for the equatorial and polar regions with temperature and salinity transport between them. Let the equatorial (respectively, polar) box contain fluid at temperature $T_{e}\left(T_{p}\right)$ and salinity $S_{e}\left(S_{p}\right)$. 'Toy model' equations for the exchange of temperature and salinity between the boxes were introduced by Stommel (1961):

$$
\dot{T}=\eta_{1}-T(1+|T-S|), \quad \dot{S}=\eta_{2}-S\left(\eta_{3}+|T-S|\right)
$$

where $T=T_{e}-T_{p}$ and $S=S_{e}-S_{p}$, the parameters $\eta_{1}$ and $\eta_{2}$ measure the strengths of the thermal and freshwater forcings respectively, and $\eta_{3}<1$ is the ratio of relaxation times of temperature and salinity (a Lewis number). This model robustly captures the bistability but is clearly hugely over-simplified. Yet the bistability 
persists as the model is refined, and this allows (at least in theory) the formation of fronts and pulses. With the aid of some additional spatial inhomogeneity to allow pinning, localized states could be supported. At the very least, theoretical opportunities exist to develop spatially-extended versions of (3.1) and, for example, probe the statistics of fluctuations that might be useful in diagnosing how close to the bistability regime, or indeed to the point $L_{+}$(see figure [6) the MOC currently is.

\section{(c) Desertification}

Surprisingly similar considerations apply to models that have been proposed for the propagation of vegetation patterns, and the reverse process, desertification (von Hardenberg et al 2001; Meron et al 2007), for example the pair of dimensionless PDEs

$$
\begin{aligned}
n_{t} & =\frac{\gamma w}{1+\sigma w} n-n^{2}-\mu n+\nabla^{2} n \\
w_{t} & =p-(1-\rho n) w-w^{2} n+\delta \nabla^{2}(w-\beta n),
\end{aligned}
$$

where $n(x, y, t)$ and $w(x, y, t)$ are the densities of biomass and soil water, respectively, and $\gamma, \sigma, \mu, p, \rho, \delta$ and $\beta$ are non-negative parameters. The terms on the righthand-side of (3.2) describe, in order, plant growth, saturation as biomass reaches the soil carrying capacity, mortality and predation, and reproductive spread. The terms in 3.3 similarly represent precipitation, loss due to evaporation (notice that $\rho>0$ indicates that vegetation inhibits evaporation), uptake of water by plants, and diffusion of water through the soil, accounting for the suction of water by plant roots.

A realistically large value for $\delta$ of around 100 leads to a Turing instability in which vegetated (patterned) states appear in addition to the trivial solution $n=0$, $w=p$ corresponding to desert. In the low precipitation $\left(p_{0}<p<p_{c}\right)$ regime, denoted arid on figure 6(b), such a model exhibits bistability between states of arid desert (the horizontal line marked $\mathcal{B}$ ) and (almost) periodic vegetation spots. The inset in figure 6(b) indicates the hysteresis loop: a spot pattern of vegetation cannot be sustained when the precipitation $p$ falls below $p_{0}$ and the system makes an abrupt transition to the desert state. Desert persists as $p$ is then increased up to $p_{c}$ at which point the desert state becomes unstable to uniform vegetation (the line denoted $\mathcal{V}$ in the main part of figure $6 \mathrm{~b}$ ). $\mathcal{V}$ becomes unstable at $p_{1}$ (numerical investigations indicate that this instability is subcritical) to patterned states: spots, then labyrithine stripes, then a uniform vegetation pattern with holes, as $p$ increases further.

This general behaviour suggests that qualitative aspects of the formation and dynamics of localized vegetation patches, as observed in arid enviroments such as the Negev desert (Meron et al 2007) may be used as diagnostic tests for proximity to sudden desertification. The challenge is to make this a quantitative method.

\section{Conclusions}

In section 2 of this review I presented a thumbnail sketch of the current state of our understanding of localized states, concentrating almost entirely on the 1D 
case. It should be pointed out that progress is, slowly but surely, being made in 2D and 3D as well, see for example the papers by Lloyd et al (2008), Lloyd \& Sandstede (2009) and Taylor \& Dawes (2009) who discuss steady localized states, and Bode et al. (2002) who discuss localized states that travel horizontally and interact strongly through collisions. In section 3 I speculated about the usefulness of the present theory for understanding and predicting various abrupt changes in particular physical systems. I will finish with brief remarks on coherent structures more generally.

Although it appears difficult to give a precise definition of a coherent structure, the term is frequently used in describing more-or-less fully developed turbulent flows. In such flows, large, long-lived eddies are prominent flow features. How might the theory of localized states be useful in this setting? Firstly, of course, one is dealing with vortical rather than density-like coherent objects. But nevertheless one might be able to exploit a separation of timescales, along with a suitable set of 'effective coordinates' for the evolution of the scale and position of these relatively large, slow-moving structures moving over a more rapidly-evolving background flow field that can be characterised through averaged properties. Such a reduction, at least locally in time, might help describe the emergence and destruction of coherent structures and thereby offer insights into fully-developed turbulence. Elements of such an approach, with substantial physical intuition but without a detailed mathematical analysis, have been employed already in nonlinear optics (Akhmediev \& Ankiewicz 2005). An easier place to begin pursuing this kind of programme might be spatiotemporally complicated flows such as 'spiral defect chaos' where again the dynamics is driven by (vertical) vorticity (Morris et al 1993). Extensions of the 2D Swift-Hohenberg equation to take account of vertical vorticity naturally have a similar form to the large-scale density-like mode case (Dawes 2008) since the vorticity equation is of nonlinear diffusion type, and is neutrally stable at zero wavenumber.

The author would like to thank Helmut Brand, John Burke, Alan Champneys, Steve Cox, Steve Houghton, Edgar Knobloch, Gregory Kozyreff, David Lloyd, Paul Matthews, Michael Proctor, Hans-George Purwins, Reinhardt Richter, Hermann Riecke, Bjorn Sandstede, Chris Taylor and Thomas Wagenknecht for useful discussions over several years on a wide range of topics related to this subject. The author also gratefully acknowledges financial support from the Royal Society through a University Research Fellowship.

\section{References}

N. Akhmediev \& A. Ankiewicz (eds) Dissipative Solitons. Lect. Notes in Physics 661, Springer, Berlin (2005)

M. Beck, J. Knobloch, D. Lloyd, B. Sandstede \& T. Wagenknecht, Snakes, ladders and isolas of localized patterns. SIAM J. Math. Anal. 41, 936-972 (2009)

A. Bergeon, J. Burke, E. Knobloch \& I. Mercader, Eckhaus instability and homoclinic snaking. Phys. Rev. E 78, 046201 (2008)

N.L. Bindoff, J. Willebrand, V. Artale, A, Cazenave, J. Gregory, S. Gulev, K. Hanawa, C. Le Qur, S. Levitus, Y. Nojiri, C.K. Shum, L.D. Talley \& A. Unnikrishnan, Observations: Oceanic Climate Change and Sea Level. In: Climate Change 200\%: The Physical Science Basis. Contribution of Working Group I to the Fourth Assessment Report of the Intergovernmental Panel on Climate Change [Solomon, S., D. Qin, M. Manning, Z. Chen, M. Marquis, K.B. Averyt, M. Tignor and H.L. Miller (eds.)]. Cambridge University Press, Cambridge, United Kingdom and New York, NY, USA. (2007) 
M. Bode, A.W. Liehr, C.P. Schenk \& H.-G. Purwins, Interaction of dissipative solitons: particle-like behaviour of localized structures in a three-component reaction-diffusion system. Physica 161D, 45-66 (2002)

S.J. Chapman and G. Kozyreff Exponential asymptotics of localized patterns and snaking bifurcation diagrams. Physica D 238, 319-354 (2009)

P. Coullet, C. Riera and C. Tresser, Stable static localized structures in one dimension. Phys. Rev. Lett. 84, 3069-3072 (2000)

M. Cross \& P.C. Hohenberg, Pattern formation outside of equilibrium Rev. Mod. Phys., 65, 851-1112 (1993)

J.H.P. Dawes, Localized convection cells in the presence of a vertical magnetic field. $J$. Fluid Mech. 570, 385-406 (2007)

J.H.P. Dawes, Localized pattern formation with a large-scale mode: slanted snaking. SIAM J. Appl. Dyn. Syst. 7, 186-206 (2008)

J.H.P. Dawes, Modulated and localized states in a finite domain. SIAM J. Appl. Dyn. Syst., 8, 909-930 (2009)

J.H.P. Dawes \& S. Lilley, Localized states in a model of pattern formation in a vertically vibrated layer. SIAM J. Appl. Dyn. Syst. To appear (2010)

E. J. Doedel, AUTO07p: continuation and bifurcation software for ordinary differential equations. Technical report, Concordia University (2007)

R.B. Hoyle, Pattern Formation: an introduction to methods. CUP (2006)

G.W. Hunt, Peletier M.A., Champneys A.R., Woods P.D., Ahmer Wadee M., Budd C.J. and Lord G.J., Cellular buckling in long structures. Nonlinear Dynamics 21, 3-29 (2000)

G. Iooss \& M.C. Pérouème, Periodic homoclinic solutions in reversible $1: 1$ resonance vector fields. J. Diff. Eq. 102, 62-88 (1993)

S.E. Huisman, M. den Toom, H.A. Dijkstra \& S. Drijfhout, An indicator of the multiple equilibria regime of the Atlantic Meridional Overturning Circulation. J. Geophys. Oceanography To appear (2009)

B. Launder \& J.M.T. Thompson, eds Geoscale Engineering to avert Dangerous Climate Change. Theme Issue of Phil. Trans. R. Soc. A 366, No. 1882 (2008)

O. Lioubashevski, Y. Hamiel, A. Agnon, Z. Reches \& J. Fineberg, Oscillons and propagating solitary waves in a vertically vibrated colloidal suspension. Phys. Rev. Lett. 83, 3190-3193 (1999)

D.J.B. Lloyd, B. Sandstede, D. Avitabile \& A.R. Champneys, Localized hexagon patterns of the planar Swift-Hohenberg equation. SIAM J. Appl. Dyn. Syst. 7, 1049 (2008)

D. Lloyd \& B. Sandstede, Localized radial solutions of the Swift-Hohenberg equation. Nonlinearity 22, 485-524 (2009)

P. C. Matthews \& S. M. Cox, Pattern formation with a conservation law. Nonlinearity 13, 1293-1320 (2000)

E. Meron, H. Yizhaq \& E. Gilad, Localized structures in dryland vegetation: forms and functions. Chaos 17, 037109 (2007)

S.W. Morris, E. Bodenschatz, D.S. Cannell \& G. Ahlers, Spiral defect chaos in large aspect ratio Rayleigh-Bénard convection. Phys. Rev. Lett. 71, 2026-2029 (1993)

Y. Pomeau, Front motion, metastability and subcritical bifurcations in hydrodynamics. Physica 23D 3-11 (1986)

M. Potier-Ferry, Amplitude modulation, phase modulation and localization of buckling patterns. In Proceedings of the IUTAM Symposium Collapse: the buckling of structures in theory and practice, eds J.M.T. Thompson and G.W. Hunt. Cambridge University Press (1982)

H.-G. Purwins, H.U. Bödeker and A.W. Liehr, Dissipative solitons in reaction-diffusion systems. In Akhmediev \& Ankiewicz (2005), 267-308 
Lord Rayleigh, On convection currents in a horizontal layer of fluid when the higher temperature is on the under side. Phil. Mag. 32, 529-543 (1916)

R. Richter \& I.V. Barashenkov, Two-dimensional solitons on the surface of magnetic fluids. Phys. Rev. Lett. 94, 184503 (2005)

H. Sakaguchi \& H.R. Brand, Stable localized solutions of arbitrary length for the quintic Swift-Hohenberg equation. Physica D 97, 274-285 (1996)

T.M. Schneider, D. Marinc \& B. Eckhardt, Localization in plane Couette edge dynamics. In B. Eckhardt (ed.), Advances in Turbulence XII, Springer Proceedings in Physics 132, 83-85 (2009)

T.M. Schneider, D. Marinc \& B. Eckhardt, Localized edge states nucleate turbulence in extended plane Couette cells. J. Fluid Mech. In press (2010)

T.M. Schneider, J.F. Gibson \& J. Burke, Snakes and ladders: localized solutions of plane Couette flow. Preprint (2010)

H. Stommel, Thermohaline convection with two stable regimes of flow. Tellus 13, 224-230 (1961)

J.B. Swift \& P.C. Hohenberg, Hydrodynamic fluctuations at the convective instability. Phys. Rev. A 15, 319-328 (1977)

C.R.N. Taylor \& J.H.P. Dawes, Snaking and isolas of localized states in bistable discrete lattices. Preprint (2009)

L.S. Tsimring \& I.S. Aranson, Localized and cellular patterns in a vibrated granular layer. Phys. Rev. Lett. 79, 213-216 (1997)

A.M. Turing, The chemical basis of morphogenesis. Phil. Trans. Roy. Soc. B 237, 37-72, 1952

P.B. Umbanhowar, F. Melo \& H.L. Swinney, Localized excitations in a vertically vibrated granular layer. Nature 382, 793-796 (1996)

J. von Hardenberg \& E. Meron \& M. Shachak \& Y. Zarmi, Diversity of vegetation patterns and desertification. Phys. Rev. Lett. 87, 198101 (2001)

F. Waleffe, On a self-sustaining process in shear flows. Phys. Fluids 9, 883-900 (1997)

J.A. Whitehead, Multiple states in doubly driven flow. Physica 97D, 311-321 (1996)

P.D. Woods and A.R. Champneys, Heteroclinic tangles and homoclinic snaking in the unfolding of a degenerate reversible Hamiltonian-Hopf bifurcation. Physica D 129, 147-170 (1999) 